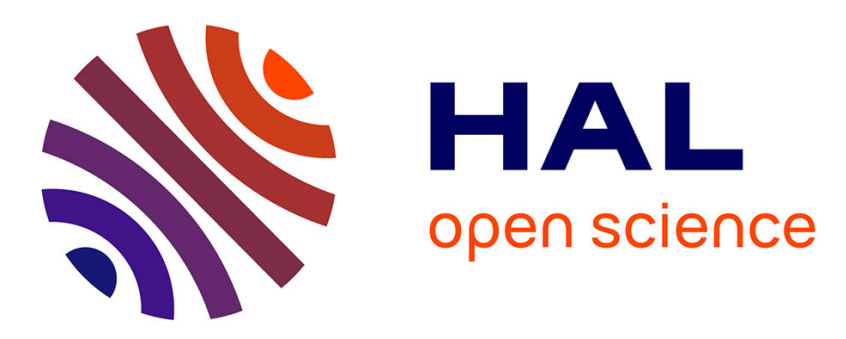

\title{
Disorder-induced phase transition in Dirac systems beyond the linear approximation
}

\author{
Sergey S. Krishtopenko, Mauro Antezza, Frederic Teppe
}

\section{To cite this version:}

Sergey S. Krishtopenko, Mauro Antezza, Frederic Teppe. Disorder-induced phase transition in Dirac systems beyond the linear approximation. Physical Review B, 2020, 101, pp.205424. 10.1103/PhysRevB.101.205424 . hal-02617155

\section{HAL Id: hal-02617155 \\ https://hal.science/hal-02617155}

Submitted on 14 Oct 2020

HAL is a multi-disciplinary open access archive for the deposit and dissemination of scientific research documents, whether they are published or not. The documents may come from teaching and research institutions in France or abroad, or from public or private research centers.
L'archive ouverte pluridisciplinaire $\mathbf{H A L}$, est destinée au dépôt et à la diffusion de documents scientifiques de niveau recherche, publiés ou non, émanant des établissements d'enseignement et de recherche français ou étrangers, des laboratoires publics ou privés. 


\title{
Disorder-induced phase transition in Dirac systems beyond the linear approximation
}

\author{
Sergey S. Krishtopenko $\odot,{ }^{1,2}$ Mauro Antezza $\odot,{ }^{1,3}$ and Frédéric Teppe ${ }^{1,2, *}$ \\ ${ }^{1}$ Laboratoire Charles Coulomb (L2C), UMR 5221 CNRS-Université de Montpellier, F-34095 Montpellier, France \\ ${ }^{2}$ CENTERA Laboratories, Institute of High Pressure Physics, Polish Academy of Sciences, PL-01-142 Warsaw, Poland \\ ${ }^{3}$ Institut Universitaire de France, 1 rue Descartes, F-75231 Paris Cedex 05, France
}

(Received 15 December 2019; accepted 6 May 2020; published 22 May 2020)

\begin{abstract}
By using the self-consistent Born approximation, we investigate disorder effect induced by the short-range impurities on the band gap in two-dimensional Dirac systems with the higher order terms in momentum. Starting from the Bernevig-Hughes-Zhang (BHZ) model, we calculate the density of states as a function of the disorder strength. We show that due to quadratic corrections to the Dirac Hamiltonian, the band gap is always affected by the disorder even if the system is gapless in the clean limit. Finally, we explore the disorder effects by using an advanced effective Hamiltonian describing the side maxima of the valence subband in HgTe quantum wells. We show that the band gap and disorder-induced topological phase transition in the real structures may differ significantly from those predicted within the BHZ model.
\end{abstract}

DOI: 10.1103/PhysRevB.101.205424

\section{INTRODUCTION}

The rise of graphene [1] has paved the way to the intensive investigation of Dirac fermions in condensed matter [2]. Since then, Dirac fermion physics has also been analyzed in many other two-dimensional (2D) systems [3]. From a general point of view, the presence of the massless Dirac cones is protected against any single-particle and many-body perturbations, at least as long as the interaction does not lead to a spontaneous breaking of symmetry [4-9]. The latter means that disorder cannot open the band gap in the massless Dirac model with only the linear terms in momentum [10-15].

Relatively less attention has been devoted to the study of disorder effects in the massive Dirac model [16-18]. The analysis within the self-consistent Born approximation (SCBA) reveals a band-gap closing above a threshold of the disorder strength (see also Fig. 1). Note that the strong disorder may also produce the onset of midgap impurity-induced states in highly disordered massive Dirac models [19-21], which needs a $t$-matrix approach (beyond the SCBA scheme) to be revealed.

Many 2D systems, however, host Dirac fermions at small momentum only, while their description requires the terms beyond the linear approximation. Prominent examples are the surface states of three-dimensional topological insulators (3D TIs) [22-24] and their films [25-27]. Other 2D systems are $\mathrm{HgTe} / \mathrm{CdHgTe}$ [28-30] and three-layer InAs/GaSb quantum wells (QWs) [31-33]. All of them are described by the Bernevig-Hughes-Zhang (BHZ) Hamiltonian [28], in which the quadratic corrections to the Dirac model allow for the proper characterization of the topological states [34].

The role of disorder beyond the linear approximation became yet more complicated after numerical simulations of Li et al. [35]. By using a tight-binding version of the BHZ Hamiltonian, they have found that disorder may induce a

*frederic.teppe@umontpellier.fr novel phase with a quantized conductance called a topological Anderson insulator (TAI) [35]. Later, Groth et al. [36] have shown that the formation of a TAI is caused by the quadratic terms $\propto k^{2} \sigma_{z}$ in the BHZ Hamiltonian, which are absent for graphene even beyond the linear approximation [2]. Moreover, it was shown that contrary to the name "topological Anderson insulator," such weak-disorder topological transition is not an Anderson transition at all, and it can be treated within the SCBA [36-38]. Although the mentioned works [35-38] are based on the tight-binding calculations on the square lattice with the constant $a$ (typically $a=5 \mathrm{~nm}$ [35-38]), they indicate that the disorder effects in the BHZ Hamiltonian may differ significantly from those known in the linear Dirac model.

In this work, we investigate how disorder changes the band gap in Dirac systems beyond the linear approximation. By using the SCBA, we directly calculate the density of states (DOS) within the continuous BHZ Hamiltonian and more advanced model [39] describing the side maxima of the valence band in $\mathrm{HgTe} \mathrm{QWs}$. Our results univocally demonstrate a crucial role of the high-order terms in the disorder effects.

\section{TWO-BAND BHZ MODEL AND SCBA}

The low-energy BHZ Hamiltonian has the form

$$
H_{2 \mathrm{D}}(\mathbf{k})=\left(\begin{array}{cc}
H_{\mathrm{BHZ}}(\mathbf{k}) & 0 \\
0 & H_{\mathrm{BHZ}}^{*}(-\mathbf{k})
\end{array}\right),
$$

where the asterisk stands for complex conjugation, $\mathbf{k}=$ $\left(k_{x}, k_{y}\right)$ is the momentum in the plane, and $H_{\mathrm{BHZ}}(\mathbf{k})=\epsilon_{k} \mathbf{I}_{2}+$ $d_{a}(\mathbf{k}) \sigma_{a}$. Here, $\mathbf{I}_{2}$ is a $2 \times 2$ unit matrix, $\sigma_{a}$ are the Pauli matrices, $\epsilon_{k}=C-D k^{2}, d_{1}(\mathbf{k})=-A k_{x}, d_{2}(\mathbf{k})=-A k_{y}, d_{3}(k)=$ $M-B k^{2}$, and $k^{2}=k_{x}^{2}+k_{y}^{2}$. In the QWs case, the mass parameter $M$ describes inversion between the electronlike $E 1$ and holelike $H 1$ subbands: $M>0$ corresponds to a trivial state, while $M<0$ for a quantum spin Hall insulator (QSHI) state [28]. For the surface states of 3D TIs, nonvanishing $M$ conforms to the gap opened due to the tunnel coupling 
between the opposite surfaces in the thin films [25-27]. A block-diagonal form of $H_{2 \mathrm{D}}(\mathbf{k})$ in Eq. (1) (cf. Refs. [40,41]) allows one to focus on the upper block only, while the calculations for the lower block are performed in the same way.

In order to calculate the DOS in the presence of disorder, we add the random impurity potential to $H_{\mathrm{BHZ}}(\mathbf{k})$ :

$$
V_{\mathrm{imp}}(\mathbf{r})=\sum_{j} v\left(\mathbf{r}-\mathbf{R}_{j}\right), \quad v(\mathbf{r})=\int \frac{d^{2} \mathbf{q}}{(2 \pi)^{2}} \tilde{v}(\mathbf{q}) e^{i \mathbf{q} \cdot \mathbf{r}},
$$

where $R_{j}$ denotes the position of impurities and $v(\mathbf{r})$ is the potential of an individual impurity, which is assumed to be isotropic, i.e., $\tilde{v}(\mathbf{q})=\tilde{v}(q)$ with $|\mathbf{q}|=q$. Then, we start from the Dyson equation in the momentum representation for the disorder-averaged Green's function $\hat{G}(\mathbf{k}, \varepsilon)$ and the selfenergy matrix $\hat{\Sigma}(\mathbf{k}, \varepsilon)$ considered in the SCBA, and illuminate the dependence on the direction of $\mathbf{k}$ by applying a unitary transformation such as $\tilde{H}_{\mathrm{BHZ}}(k)=U\left(\theta_{\mathbf{k}}\right) H_{\mathrm{BHZ}}(\mathbf{k}) U\left(\theta_{\mathbf{k}}\right)^{-1}$. After some calculations provided in the Supplemental Material [42], we get the following self-consistent equations:

$$
\begin{aligned}
\hat{\Sigma}(k, \varepsilon) & =n_{i} \int_{0}^{K_{c}} \frac{k^{\prime} d k^{\prime}}{2 \pi}\left(\begin{array}{cc}
V_{0}\left(k, k^{\prime}\right)^{2} G_{11}^{\prime} & V_{-1}\left(k, k^{\prime}\right)^{2} G_{12}^{\prime} \\
V_{+1}\left(k, k^{\prime}\right)^{2} G_{21}^{\prime} & V_{0}\left(k, k^{\prime}\right)^{2} G_{22}^{\prime}
\end{array}\right), \\
V_{n}\left(k, k^{\prime}\right)^{2} & =\int_{0}^{2 \pi} \frac{d \theta}{2 \pi}\left|\tilde{v}\left(\mathbf{k}-\mathbf{k}^{\prime}\right)\right|^{2} \cos n \theta
\end{aligned}
$$

where $n_{i}$ is the concentration of impurities, and $G_{i j}^{\prime} \equiv$ $G_{i j}\left(k^{\prime}, \varepsilon\right)$ are the components of the Green's function $\hat{G}(k, \varepsilon)=\left[\varepsilon-\tilde{H}_{\mathrm{BHZ}}(k)-\hat{\Sigma}(k, \varepsilon)\right]^{-1}$. In Eqs. (3), we introduce a cut-off wave vector $K_{c}=\pi / a_{0}$ (where $a_{0}$ is the lattice constant), corresponding to the size of the Brillouin zone (cf. Refs. [16-18]). Once the Green's function is known, the DOS can be calculated as

$$
D(\varepsilon)=-\frac{g_{S}}{\pi} \int_{0}^{K_{c}} \frac{k d k}{2 \pi} \operatorname{Im}\{\operatorname{Tr}[\hat{G}(k, \varepsilon+i 0)]\},
$$

where the factor $g_{S}=2$ takes into account the contribution from the lower block in Eq. (1).

To proceed further, we assume $\tilde{v}(q)=u_{0}$, which corresponds to the disorder formed by the short-range impurities [16-18]. In this case, the self-energy matrix is independent of $k$ and has the form $\hat{\Sigma}(\varepsilon)=\Sigma_{0}(\varepsilon) \mathbf{I}_{2}+\Sigma_{z}(\varepsilon) \sigma_{z}$. Under these conditions, the set in Eq. (3) is written as

$$
\Sigma_{0}=\frac{W^{2}}{4 \pi} \int_{0}^{K_{c}^{2}} \frac{X+D x}{\Lambda(x, \varepsilon)} d x, \quad \Sigma_{z}=\frac{W^{2}}{4 \pi} \int_{0}^{K_{c}^{2}} \frac{Y-B x}{\Lambda(x, \varepsilon)} d x,
$$

where $W$ is a disorder strength defined as $W^{2}=n_{i} u_{0}^{2}$, $X(\varepsilon)=\varepsilon-C-\Sigma_{0}(\varepsilon), Y(\varepsilon)=M+\Sigma_{z}(\varepsilon)$, and $\Lambda(x, \varepsilon)=$ $\left(D^{2}-B^{2}\right) x^{2}+\left(2 B Y+2 D X-A^{2}\right) x+X^{2}-Y^{2}$. Note that the above integrals are calculated analytically [42], transforming Eq. (5) into the set of algebraic equations numerically solved by simple iterations.

First, we consider the case of the linear Dirac model, corresponding to zero values of $B$ and $D$. As shown in Fig. 1, the band gap of the massive Dirac fermions decreases by increasing $W$ until it vanishes above a critical value $W^{*}$. Such behavior was also investigated previously [16-18]. For the gapless system, disorder does not open a band gap as was
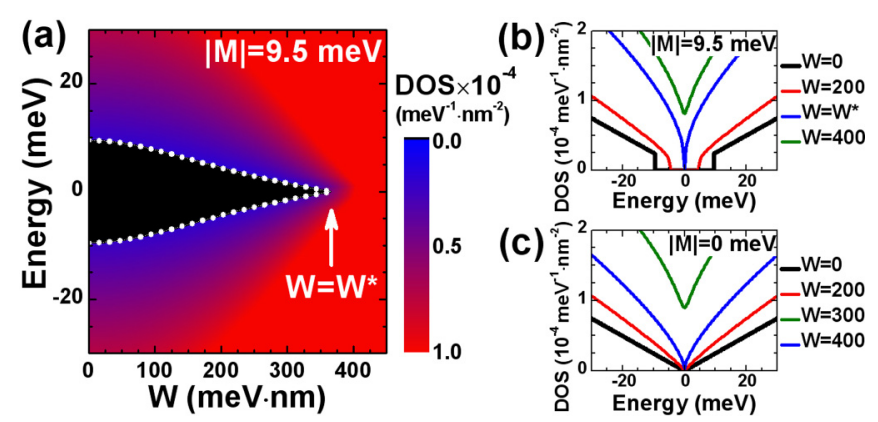

FIG. 1. (a) Color map of the DOS as a function of the disorder strength $W$ for the massive Dirac fermions with the energy $E=$ $\pm \sqrt{M^{2}+A^{2} k^{2}}$, where $|M|=9.5 \mathrm{meV}$ and $A=358 \mathrm{meV} \mathrm{nm}$. The white curves represent the band edges with vanishing DOS described by Eq. (6). (b),(c) The DOS at different values of $W$ for $|M|=9.5$ $\mathrm{meV}$ and $|M|=0 \mathrm{meV}$.

shown before for graphene [10-15]. This is also seen from Eq. (5), as $\Sigma_{z}(\varepsilon)=0$ is the self-consistent solution at $M=0$ and $B=D=0$. Note that the changes of DOS with $W$ are independent of the sign of $M$ in the linear model.

The situation changes dramatically if we consider the square terms $\propto B k^{2} \sigma_{z}$. Although we also include the terms $\propto D k^{2} \mathbf{I}_{2}$ representing the electron-hole asymmetry, the DOS evolution remains qualitatively the same even as for $D=0$. Further, we focus on $\mathrm{HgTe}$ QWs, which require nonvanishing $D$ for their description $[39,43]$. As the band ordering in HgTe QWs is affected by hydrostatic pressure [44], temperature $[45,46]$, and strain $[47,48]$, we note to consider $\mathrm{HgTe} / \mathrm{Cd}_{0.7} \mathrm{Hg}_{0.3} \mathrm{Te} \mathrm{QWs}$ grown on (001) CdTe buffer at zero temperature and pressure [44].

Figure 2 shows the evolution of DOS with the disorder strength $W$ for the HgTe QWs of different QW width. As is seen, in contrast to the linear model, now the band-gap evolution strongly depends on the sign of $M$. If $M>0$, the band gap decreases with $W$ and vanishes at a critical value $W^{*}$, and then it is reopened again at $W>W^{*}$. Such behavior represents the disorder-induced topological phase transition previously discovered in the tight-binding calculations $[35,36]$. Let us now analyze it within the continuous BHZ model.

Since the finite DOS is associated with a finite imaginary part of the functions $\Sigma_{0}(\varepsilon)$ and $\Sigma_{z}(\varepsilon)$, the band-gap region is characterized by the solution of Eq. (5) with purely real quantities $\bar{\Sigma}_{0}(\varepsilon)$ and $\bar{\Sigma}_{z}(\varepsilon)$. The band edges can be obtained by solving the following equations:

$$
\bar{X}(\varepsilon)=\bar{Y}(\varepsilon), \quad \bar{X}(\varepsilon)=-\bar{Y}(\varepsilon),
$$

where the overbar stresses the values found on the set of real numbers. As seen from Fig. 2, two curves described by Eq. (6) cross at the transition point $W=W^{*}$, where $\bar{Y}(\varepsilon)$ changes the sign. As shown by Groth et al. [36], $\bar{Y}(\varepsilon)$ has a meaning of the renormalized topological mass and its negative sign corresponds to the TAI state. We note that the disorderinduced phase transition at $M>0$ in Fig. 2 is caused by the negative values of $B$ in $\mathrm{HgTe}$ QWs $[28,41,43]$, resulting in $\bar{\Sigma}_{z}(\varepsilon)<0$. In the systems with $B>0$, such transition arises at $M<0$. 

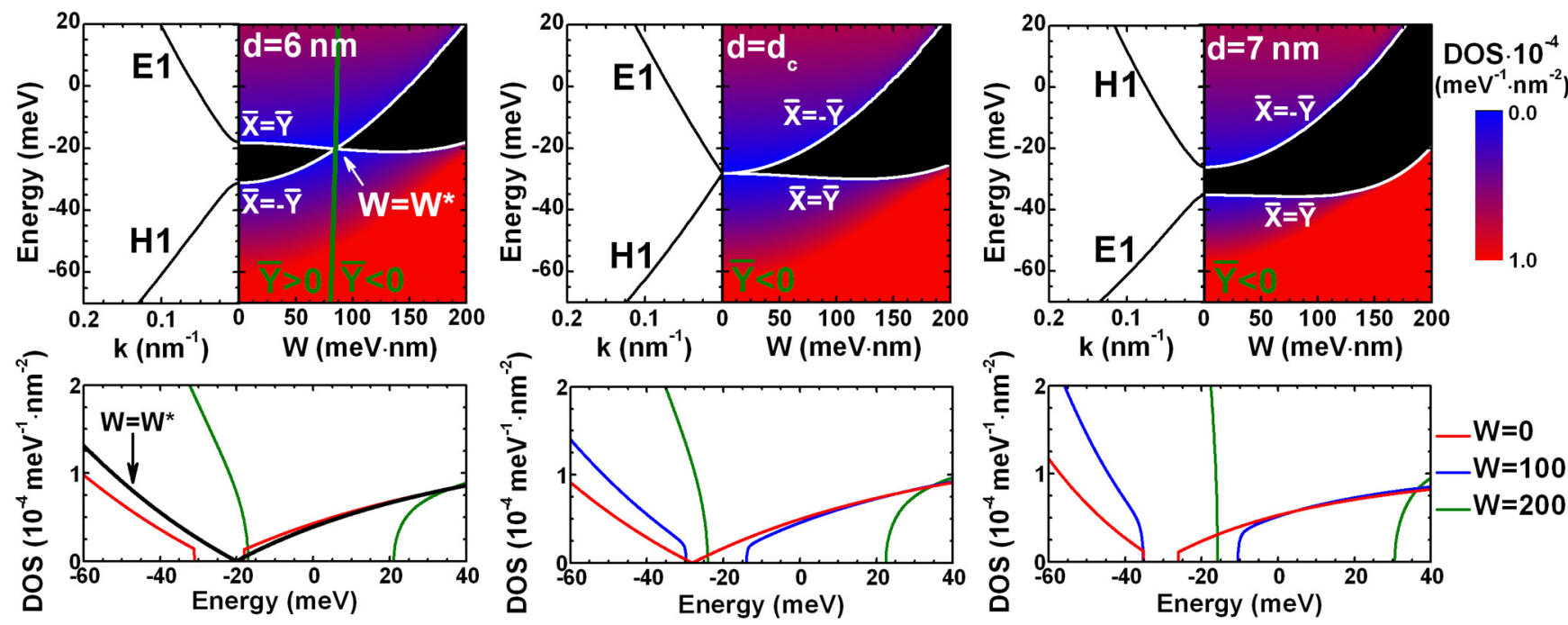

FIG. 2. Band structure and color map of the DOS as a function of the disorder strength $W$ calculated in the two-band BHZ model for HgTe QW at different QW width: $d=6 \mathrm{~nm}(M>0), d=d_{c}(M=0)$, and $d=7 \mathrm{~nm}(M<0)$. The band parameters are provided in the Supplemental Material [42]. The white curves represent the edges of the area with the vanishing DOS described by Eq. (6). The green curve is found from $\bar{Y}(\varepsilon)=0$. The bottom panels show the DOS at several values of $W$.

As mentioned above, the disorder does not open the gap for the linear massless Dirac fermions. Figure 2 demonstrates that due to the square terms $\propto B k^{2} \sigma_{z}$, the gapless state becomes a critical state with $W^{*}=0$, and the band gap is now affected by the disorder. Interestingly, one may conclude that since the surface states of 3D TIs are described by the BHZ Hamiltonian, they are not robust to the surface disorder. However, the parameters $M$ and $B$ are not independent for 3D TIs [25-27]. In the absence of the tunnel coupling between the opposite surfaces, $M=0$ but $B$ vanishes as well $[25,26]$. The latter prevents the band-gap opening by the disorder.

We have considered a role of the square terms in the disorder-induced topological phase transition in Dirac systems. Further, we investigate how the higher-order terms beyond the BHZ model affect the band gap in the real structures. These terms are crucial for the side maxima (SM) of the valence subband in $\mathrm{HgTe}$ QWs $[39,44]$

\section{FOUR-BAND 2D MODEL AND SCBA}

The advanced Hamiltonian for $\mathrm{HgTe}$ QWs including the second electronlike $E 2$ and holelike $H 2$ subbands is written as

$$
H_{2 \mathrm{D}}(\mathbf{k})=\left(\begin{array}{cc}
H_{4 \times 4}(\mathbf{k}) & 0 \\
0 & H_{4 \times 4}^{*}(-\mathbf{k})
\end{array}\right)
$$

with the blocks $H_{4 \times 4}(\mathbf{k})$ and $H_{4 \times 4}^{*}(-\mathbf{k})$ defined as

$$
H_{4 \times 4}(\mathbf{k})=\left(\begin{array}{cccc}
\epsilon_{\mathbf{k}}+d_{3}(\mathbf{k}) & -A k_{+} & R_{1} k_{-}^{2} & S_{0} k_{-} \\
-A k_{-} & \epsilon_{\mathbf{k}}-d_{3}(\mathbf{k}) & 0 & R_{2} k_{-}^{2} \\
R_{1} k_{+}^{2} & 0 & \epsilon_{H 2}(\mathbf{k}) & A_{2} k_{+} \\
S_{0} k_{+} & R_{2} k_{+}^{2} & A_{2} k_{-} & \epsilon_{E 2}(\mathbf{k})
\end{array}\right),
$$

where $\epsilon_{E 2}(\mathbf{k})=C+M+\Delta_{E 1 E 2}+B_{E 2}\left(k_{x}^{2}+k_{y}^{2}\right), \epsilon_{H 2}(\mathbf{k})=$ $C-M-\Delta_{H 1 H 2}+B_{H 2}\left(k_{x}^{2}+k_{y}^{2}\right)$, and $\Delta_{E 1 E 2}$ and $\Delta_{H 1 H 2}$ are the gaps between the $E 1$ and $E 2$ subbands and the $H 1$ and $H 2$ subbands, respectively [39].
Then, with a unitary transformation such as $\tilde{H}_{4 \times 4}(k)=$ $V\left(\theta_{\mathbf{k}}\right) H_{4 \times 4}(\mathbf{k}) V\left(\theta_{\mathbf{k}}\right)^{-1}$, the self-energy matrix $\hat{\Sigma}_{4 \times 4}(k, \varepsilon)$ in the SCBA has the form [42]

$$
\begin{aligned}
\hat{\Sigma}_{4 \times 4}(k, \varepsilon)= & n_{i} \int_{0}^{K_{c}} \frac{k^{\prime} d k^{\prime}}{2 \pi} \\
& \times\left(\begin{array}{cccc}
V_{0}^{2} G_{11}^{\prime} & V_{-1}^{2} G_{12}^{\prime} & V_{+2}^{2} G_{13}^{\prime} & V_{+1}^{2} G_{14}^{\prime} \\
V_{+1}^{2} G_{21}^{\prime} & V_{0}^{2} G_{22}^{\prime} & V_{+3}^{2} G_{23}^{\prime} & V_{+2}^{2} G_{24}^{\prime} \\
V_{-2}^{2} G_{31}^{\prime} & V_{-3}^{2} G_{32}^{\prime} & V_{0}^{2} G_{33}^{\prime} & V_{-1}^{2} G_{34}^{\prime} \\
V_{-1}^{2} G_{41}^{\prime} & V_{-2}^{2} G_{42}^{\prime} & V_{+1}^{2} G_{43}^{\prime} & V_{0}^{2} G_{44}^{\prime}
\end{array}\right),
\end{aligned}
$$

where $n_{i}, K_{c}$, and $V_{n}\left(k, k^{\prime}\right)^{2}$ are the same as those for Eq. (3), while $G_{i j}^{\prime} \equiv G_{i j}\left(k^{\prime}, \varepsilon\right)$ are the components of the averaged Green's function $\hat{G}(k, \varepsilon)=\left[\varepsilon-\tilde{H}_{4 \times 4}(k)-\hat{\Sigma}(k, \varepsilon)\right]^{-1}$. In the case of the short-range impurities, the self-energy matrix is diagonal and independent of $\varepsilon$ and Eq. (9) transforms into the set of algebraic equations numerically solved by an iteration procedure [42].

Figure 3 shows the DOS evolution with the disorder for the same QW widths as in Fig. 2. As is seen for the 6-nm QW, the disorder-induced phase transition at $W=W^{*}$ is still identified. However, the values of $W^{*}$ and the areas with the vanishing DOS differ significantly in two models. Particularly, the renormalized band gap in the BHZ model may even exceed the largest gap known for the HgTe QWs [47], while the four-band model predicts the lower values.

Another feature, which cannot be addressed in the BHZ model, is the evolution of the DOS associated with the SM of the top valence subband. In the clean limit, the side maxima result in the large steplike increasing of the DOS. At nonzero $W$, such steplike behavior can be also used for qualitative determination of the SM position. The white and green curves in the top panels of Fig. 3 represent the evolution of the band edges in the $\Gamma$ point and the side maxima, respectively. In 

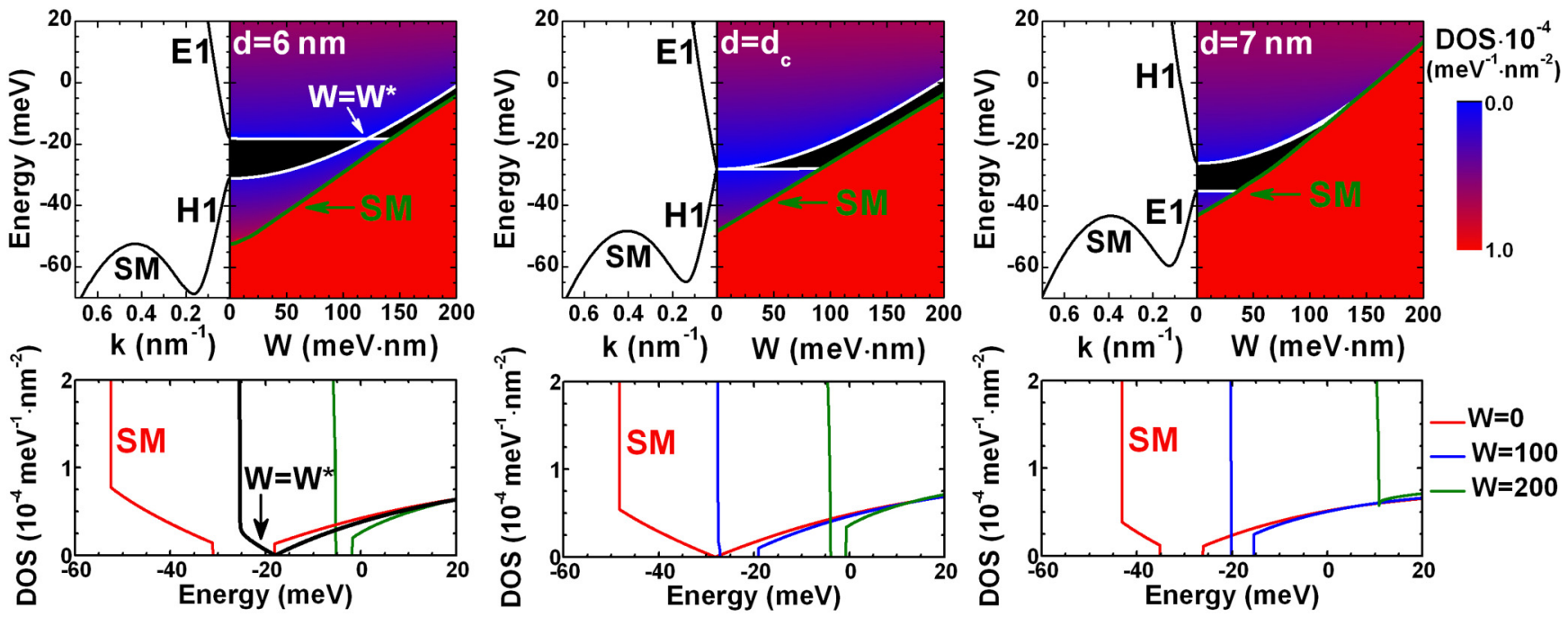

FIG. 3. Band structure and the DOS as a function of the disorder strength $W$ calculated in the four-band 2D model [39] for HgTe QW at different QW widths: $d=6 \mathrm{~nm}, d=d_{c}$, and $d=7 \mathrm{~nm}$. The band parameters are provided in the Supplemental Material [42]. The white curves represent the edges of the area with the vanishing DOS found from the numerical calculations. The green curve shows the energy edge of the side maxima identified in the DOS. The bottom panels represent the DOS at several values of $W$ for each of the QW widths.

contrast to the BHZ model, these curves can be identified only in the numerical calculations. As the SM position primarily depends on the distance between $E 2$ and $H 2$ subbands [39], its evolution with the disorder remains qualitatively the same for any values of $M$.

As is seen, the SM contribution increases with the disorder and strongly affects the area with the vanishing DOS. For the inverted HgTe QWs, the SM may result in the band-gap closing and transition into the semimetal state. The latter is clearly seen for the 9-nm-wide $\mathrm{HgTe}$ QW representing indirect-gap QSHI (see Fig. 4). Indeed, the upper boundary of the area with the vanishing DOS represents the evolution of the conduction band edge, while the lower boundary corresponds to the SM evolution. The semimetal state arises when the side maxima exceed the conduction band bottom. Note that such state also exists in the wide HgTe QWs in the clean limit $[49,50]$. Thus, the disorder may not only yield to the band inversion as explained by Groth et al. [36] but induce the semimetal state as well.

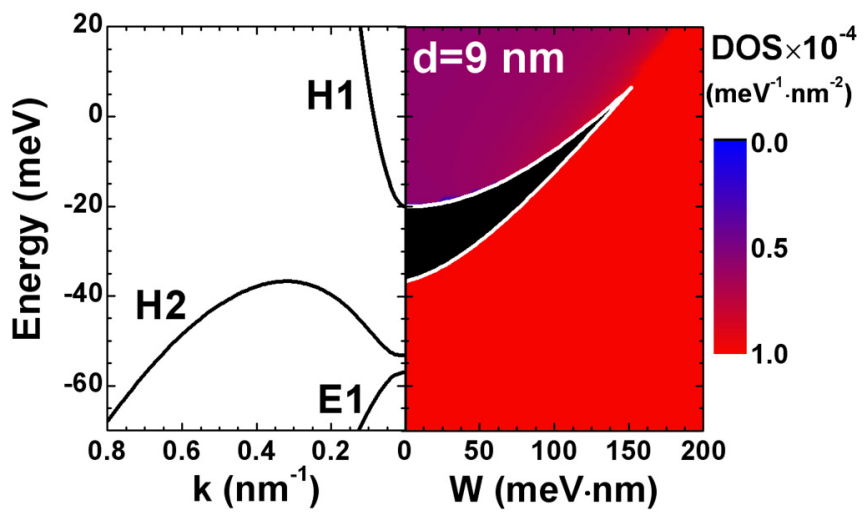

FIG. 4. Band structure and the DOS as a function of the disorder strength $W$ for the 9-nm-wide HgTe QW. The white curves represent the edges of the black area with the vanishing DOS identified in the numerical calculations.
To clarify if the disorder range in Figs 2-4 is relevant for the HgTe QWs, we calculate the electron mobility $\mu_{W}$ caused by the short-range impurities [42]. The calculations, performed in the relaxation-time approximation [51] within the BHZ model, evidence that $W<200$ corresponds to $\mu_{W}>$ $4 \mathrm{~m}^{2} / \mathrm{V}$ s for the electron concentration $n_{S}=10^{11} \mathrm{~cm}^{-2}$. This is comparable with the experimental values for $\mathrm{HgTe}$ QWs [52]. Note that the mobility evaluation in the BHZ model is quite reliable for the conduction band, since it requires the description of electronic states only in the vicinity of the Fermi level, in contrast to the self-energy matrix, which is calculated over the whole Brillouin zone.

\section{CONCLUSIONS}

We have investigated the disorder effect caused by the short-range impurities on the band gap and DOS in Dirac systems beyond the linear approximation. By using the SCBA and continuous BHZ model, we show that the quadratic corrections to the Dirac Hamiltonian always result in the band-gap renormalization even if the system is gapless in the clean limit. We have also explored the role of the high-order terms beyond the BHZ model [36] in the disorder effect in HgTe QWs. We have shown that the disorder-induced phase transition in the real structures may differ significantly from those predicted within the BHZ model. Our findings thus clearly demonstrate the invalidity of the BHZ model for quantitative description of the disorder effects in $\mathrm{HgTe}$ QWs.

\section{ACKNOWLEDGMENTS}

The authors acknowledge T. Dietl for his critical comments and discussions. This work was supported by MIPS department of Montpellier University through the "Occitanie Terahertz Platform," by the Languedoc-Roussillon region via the "Gepeto Terahertz platform," and by the French Agence Nationale pour la Recherche (Colector project). S. K. and F. T. also acknowledge the National Science Centre, Poland, (Grant No. UMO-2017/25/N/ST3/00408). 
[1] A. Geim and K. Novoselov, Nat. Mater. 6, 183 (2007).

[2] A. H. Castro Neto, F. Guinea, N. M. R. Peres, K. S. Novoselov, and A. K. Geim, Rev. Mod. Phys. 81, 109 (2009).

[3] T. O. Wehling, A. M. Black-Schaffer, and A. V. Balatsky, Adv. Phys. 63, 1 (2014).

[4] T. Appelquist, D. Nash, and L. C. R. Wijewardhana, Phys. Rev. Lett. 60, 2575 (1988).

[5] D. V. Khveshchenko, Phys. Rev. Lett. 87, 246802 (2001).

[6] O. Vafek and M. J. Case, Phys. Rev. B 77, 033410 (2008).

[7] J. Wang, H. A. Fertig, and G. Murthy, Phys. Rev. Lett. 104, 186401 (2010).

[8] V. N. Kotov, B. Uchoa, V. M. Pereira, F. Guinea, and A. H. Castro Neto, Rev. Mod. Phys. 84, 1067 (2012).

[9] C. Popovici, C. S. Fischer, and L. von Smekal, Phys. Rev. B 88, 205429 (2013).

[10] N. Shon and T. Ando, J. Phys. Soc. Jpn. 67, 2421 (1998).

[11] T. Fukuzawa, M. Koshino, and T. Ando, J. Phys. Soc. Jpn. 78, 094714 (2009).

[12] V. M. Pereira, F. Guinea, J. M. B. Lopes dos Santos, N. M. R. Peres, and A. H. Castro Neto, Phys. Rev. Lett. 96, 036801 (2006).

[13] N. M. R. Peres, F. Guinea, and A. H. Castro Neto, Phys. Rev. B 73, 125411 (2006).

[14] B. Dóra, K. Ziegler, and P. Thalmeier, Phys. Rev. B 77, 115422 (2008).

[15] B. Yu-Kuang Hu, E. H. Hwang, and S. Das Sarma, Phys. Rev. B 78, 165411 (2008).

[16] Y. Arimura and T. Ando, J. Phys. Soc. Jpn. 81, 024702 (2012).

[17] T. Ando, J. Phys. Soc. Jpn. 84, 114705 (2015).

[18] H. Rostami and E. Cappelluti, Phys. Rev. B 96, 054205 (2017).

[19] A. V. Balatsky, I. Vekhter, and J.-X. Zhu, Rev. Mod. Phys. 78, 373 (2006).

[20] J. W. González and J. Fernández-Rossier, Phys. Rev. B 86, 115327 (2012).

[21] E. V. Castro, M. P. López-Sancho, and M. A. H. Vozmediano, Phys. Rev. B 92, 085410 (2015).

[22] L. Fu and C. L. Kane, Phys. Rev. B 76, 045302 (2007).

[23] Y. Xia, D. Qian, D. Hsieh, L. Wray, A. Pal, H. Lin, A. Bansil, D. Grauer, Y. S. Hor, R. J. Cava, and M. Z. Hasan, Nat. Phys. 5, 398 (2009).

[24] H. Zhang, C.-X. Liu, X.-L. Qi, X. Dai, Z. Fang, and S.-C. Zhang, Nat. Phys. 5, 438 (2009).

[25] W.-Y. Shan, H.-Z. Lu, and S.-Q. Shen, New J. Phys. 12, 043048 (2010).

[26] H.-Z. Lu, W.-Y. Shan, W. Yao, Q. Niu, and S.-Q. Shen, Phys. Rev. B 81, 115407 (2010).

[27] C.-X. Liu, H. J. Zhang, B. Yan, X.-L. Qi, T. Frauenheim, X. Dai, Z. Fang, and S.-C. Zhang, Phys. Rev. B 81, 041307(R) (2010).

[28] B. A. Bernevig, T. L. Hughes, and S.-C. Zhang, Science 314, 1757 (2006).

[29] M. König, S. Wiedmann, C. Brüne, A. Roth, H. Buhmann, L. W. Molenkamp, X.-L. Qi, and S.-C. Zhang, Science 318, 766 (2007).

[30] B. Büttner, C. Liu, G. Tkachov, E. Novik, C. Brüne, H. Buhmann, E. Hankiewicz, P. Recher, B. Trauzettel, S. Zhang, and L. Molenkamp, Nat. Phys. 7, 418 (2011).

[31] S. S. Krishtopenko and F. Teppe, Sci. Adv. 4, eaap7529 (2018).
[32] S. S. Krishtopenko, S. Ruffenach, F. Gonzalez-Posada, G. Boissier, M. Marcinkiewicz, M. A. Fadeev, A. M. Kadykov, V. V. Rumyantsev, S. V. Morozov, V. I. Gavrilenko, C. Consejo, W. Desrat, B. Jouault, W. Knap, E. Tournié, and F. Teppe, Phys. Rev. B 97, 245419 (2018).

[33] S. S. Krishtopenko, W. Desrat, K. E. Spirin, C. Consejo, S. Ruffenach, F. Gonzalez-Posada, B. Jouault, W. Knap, K. V. Maremyanin, V. I. Gavrilenko, G. Boissier, J. Torres, M. Zaknoune, E. Tournié, and F. Teppe, Phys. Rev. B 99, 121405(R) (2019).

[34] S.-Q. Shen, W.-Y. Shan, and H.-Z. Lu, SPIN 01, 33 (2011).

[35] J. Li, R.-L. Chu, J. K. Jain, and S.-Q. Shen, Phys. Rev. Lett. 102, 136806 (2009).

[36] C. W. Groth, M. Wimmer, A. R. Akhmerov, J. Tworzydło, and C. W. J. Beenakker, Phys. Rev. Lett. 103, 196805 (2009).

[37] L. Chen, Q. Liu, X. Lin, X. Zhang, and X. Jiang, New J. Phys. 14, 043028 (2012).

[38] A. Girschik, F. Libisch, and S. Rotter, Phys. Rev. B 88, 014201 (2013).

[39] S. S. Krishtopenko and F. Teppe, Phys. Rev. B 97, 165408 (2018).

[40] M. König, H. Buhmann, L. W. Molenkamp, T. Hughes, C.-X. Liu, X.-L. Qi, and S.-C. Zhang, J. Phys. Soc. Jpn. 77, 031007 (2008).

[41] D. G. Rothe, R. W. Reinthaler, C.-X. Liu, L. W. Molenkamp, S.-C. Zhang, and E. M. Hankiewicz, New J. Phys. 12, 065012 (2010).

[42] See Supplemental Material at http://link.aps.org/supplemental/ 10.1103/PhysRevB.101.205424, which also contains Ref. [53], for any details of the SCBA within the two-band BHZ model [28] and the four-band advanced 2D Hamiltonian [39]. The mobility calculation for the short-range impurities based on the BHZ Hamiltonian, as well as the parameters for both models are also provided therein.

[43] A. M. Kadykov, S. S. Krishtopenko, B. Jouault, W. Desrat, W. Knap, S. Ruffenach, C. Consejo, J. Torres, S. V. Morozov, N. N. Mikhailov, S. A. Dvoretskii, and F. Teppe, Phys. Rev. Lett. 120, 086401 (2018).

[44] S. S. Krishtopenko, I. Yahniuk, D. B. But, V. I. Gavrilenko, W. Knap, and F. Teppe, Phys. Rev. B 94, 245402 (2016).

[45] S. Wiedmann, A. Jost, C. Thienel, C. Brüne, P. Leubner, H. Buhmann, L. W. Molenkamp, J. C. Maan, and U. Zeitler, Phys. Rev. B 91, 205311 (2015).

[46] M. Marcinkiewicz, S. Ruffenach, S. S. Krishtopenko, A. M. Kadykov, C. Consejo, D. B. But, W. Desrat, W. Knap, J. Torres, A. V. Ikonnikov, K. E. Spirin, S. V. Morozov, V. I. Gavrilenko, N. N. Mikhailov, S. A. Dvoretskii, and F. Teppe, Phys. Rev. B 96, 035405 (2017).

[47] P. Leubner, L. Lunczer, C. Brüne, H. Buhmann, and L. W. Molenkamp, Phys. Rev. Lett. 117, 086403 (2016).

[48] I. Yahniuk, S. S. Krishtopenko, G. Grabecki, B. Jouault, C. Consejo, W. Desrat, M. Majewicz, A. M. Kadykov, K. E. Spirin, V. I. Gavrilenko, N. N. Mikhailov, S. A. Dvoretsky, D. B. But, F. Teppe, J. Wrobel, G. Cywinski, S. Kret, T. Dietl, and W. Knap, npj Quantum Mater. 4, 13 (2019).

[49] Z. D. Kvon, E. B. Olshanetsky, D. A. Kozlov, N. N. Mikhailov, and S. A. Dvoretskii, JETP Lett. 87, 502 (2008). 
[50] Z. D. Kvon, E. B. Olshanetsky, E. G. Novik, D. A. Kozlov, N. N. Mikhailov, I. O. Parm, and S. A. Dvoretsky, Phys. Rev. B 83, 193304 (2011).

[51] E. H. Hwang and S. Das Sarma, Phys. Rev. B 77, 195412 (2008).
[52] G. Tkachov, C. Thienel, V. Pinneker, B. Büttner, C. Brüne, H. Buhmann, L. W. Molenkamp, and E. M. Hankiewicz, Phys. Rev. Lett. 106, 076802 (2011).

[53] S. S. Krishtopenko, W. Knap, and F. Teppe, Sci. Rep. 6, 30755 (2016). 